\title{
Análise da variação de temperatura corporal e da percepção subjetiva de esforço durante sessões de treinamento de futsal
}

\author{
Analysis of body temperature variation and subjective perception of effort during futsal training \\ sessions
}

Análisis de la variación de la temperatura corporal y la percepción subjetiva del esfuerzo durante las sesiones de entrenamiento de fútbol sala

Ben Hur Soares

ORCID: https://orcid.org/0000-0001-6762-4799 Universidade de Passo Fundo, Brasil E-mail: benhur@upf.br

Adriano Paqualotti

ORCID: https://orcid.org/0000-0001-7544-9425 Universidade de Passo Fundo, Brasil E-mail: pasqualotti@upf.br

Sergio Adriano Gomes ORCID: https://orcid.org/0000-0003-1989-852X Universidade Católica de Brasília, Brasil E-mail: congressofutsal@gmail.com

Mylena Aparecida Rodrigues Alves

ORCID: https://orcid.org/0000-0001-8614-407X Universidade Federal do Paraná, Brasil

E-mail: mylena_cg@hotmail.com

Arceni Júnior Barboza

ORCID: https://orcid.org/0000-0002-4061-4109 Associação Marauense de Futsal, Brasil E-mail: barbozaarcenijunior@gmail.com

José Roberto Soares

ORCID: https://orcid.org/0000-0002-2152-1871 Associação Marauense de Futsal, Brasil E-mail: ze.soares@hotmail.com

Vitor Gabriel Pagnussat de Freitas

ORCID: https://orcid.org/0000-0002-8404-7839 Associação Marauense de Futsal, Brasil

E-mail: vitorfreitas695@gmail.com

Guilherme Paixão

ORCID: https://orcid.org/0000-0003-2380-5613 Associação Marauense de Futsal, Brasil

E-mail: guizagoleiros@gmail.com

Rudy José Nodari Júnior

ORCID: https://orcid.org/0000-0002-8375-657X Salus Dermatoglifia, INOVALE, Brasil

Universidade do Oeste de Santa Catarina, Brasil

E-mail: rudy.nodarijunior@salusdermatoglifia.com.br

\begin{abstract}
Resumo
O esporte de alto rendimento proporciona mudanças de intensidades e causa oscilações na temperatura do atleta, bem como sensações diferentes de percepção de esforço, face às cargas externas aplicadas nos treinamentos e nas competições. O objetivo foi avaliar as variações de temperatura e da percepção subjetiva de esforço durante os treinamentos preparatórios para a Liga Gaúcha de Futsal, na temporada de 2020. A amostra foi composta por 19 jogadores de futsal do sexo masculino. Neste estudo, para se verificar o impacto das cargas externas de treino, foi mensurada a temperatura corporal e coletada a percepção subjetiva de esforço dos indivíduos da amostra, durante 15 sessões de treinamento. Observou-se uma variação, tanto na temperatura corporal dos atletas $\left(35,78^{\circ} \mathrm{C}\right.$ e $\left.36,25^{\circ} \mathrm{C}\right)$, nos diferentes momentos do treinamento, quanto na percepção subjetiva de esforço. No início, obteve-se valores médios de 3,12 $\pm 0,43$, passando para 4,79 $\pm 0,28$ e, no final, para 4,87 $\pm 0,33$. Esses resultados estatísticos apresentaram uma correlação significativa moderada entre a temperatura corporal e a percepção subjetiva de esforço durante o aquecimento. Diante desses achados, verificou-se uma relação diretamente proporcional entre essas variáveis, de
\end{abstract}


modo que o aumento da temperatura corporal eleva a percepção subjetiva de esforço. Face aos resultados dessa investigação, inferimos que a mensuração da percepção subjetiva de esforço se apresenta como uma ótima ferramenta para se quantificar e controlar as cargas de uma sessão de treino da modalidade futsal.

Palavras-chave: Futsal; Temperatura corporal; Percepção subjetiva de esforço; Covid-19; Pandemia.

\begin{abstract}
High performance sport provides changes in intensities and causes oscillations in the athlete's temperature, as well as different sensations of perceived exertion, given the external loads applied in training and competitions. Evaluate the variations in temperature and in the subjective perception of effort during preparatory training for the Gaucho Futsal League, in the 2020 season. The sample consisted of 19 male futsal players. In this study, to verify the impact of external training loads, body temperature was measured and the subjective perception of effort of the individuals in the sample was collected during 15 training sessions. A variation was observed, both in the athletes' body temperature $\left(35.78^{\circ} \mathrm{C}\right.$ and $\left.36.25^{\circ} \mathrm{C}\right)$, in the different moments of training, and in the subjective effort perception. At the beginning, mean values of $3.12 \pm 0.43$ were obtained, changing to $4.79 \pm 0.28$ and, at the end, to $4.87 \pm 0.33$. These statistical results showed a moderately significant correlation between body temperature and subjective effort perception in warming up. In view of these findings, there was a directly proportional relationship between these variables, such that the increase in body temperature raises the subjective effort perception. In view of the results of this investigation, we infer that the measurement of subjective effort perception is an excellent tool to quantify and control the loads of a training session in the futsal modality.
\end{abstract}

Keywords: Futsal; Body temperature; Subjective perception of effort; Covid-19; Pandemic.

\title{
Resumen
}

El deporte de alto rendimiento proporciona cambios de intensidades y provoca oscilaciones en la temperatura del deportista, así como diferentes sensaciones de esfuerzo percibido, dadas las cargas externas aplicadas en los entrenamientos y competiciones. El objetivo fue evaluar las variaciones de temperatura y la percepción subjetiva del esfuerzo durante los entrenamientos preparatorios para la Liga Gaúcha de Fútbol Sala, en la temporada 2020. La muestra estaba compuesta por 19 jugadores de fútbol sala masculinos. En este estudio, para comprobar el impacto de las cargas externas de entrenamiento, se midió la temperatura corporal y se recogió la percepción subjetiva del esfuerzo de los individuos de la muestra, durante 15 sesiones de entrenamiento. Se observó una variación, tanto en la temperatura corporal de $\operatorname{los}$ atletas $\left(35,78^{\circ} \mathrm{C}\right.$ y $\left.36,25^{\circ} \mathrm{C}\right)$, en diferentes momentos del entrenamiento, como en la percepción subjetiva del esfuerzo. Al principio, los valores medios eran de 3,12 $\pm 0,43$, cambiando a 4,79 $\pm 0,28$ y, al final, a 4,87 $\pm 0,33$. Estos resultados estadísticos mostraron una correlación moderadamente significativa entre la temperatura corporal y la percepción subjetiva del esfuerzo durante el calentamiento. A la vista de estos hallazgos, se verificó una relación directamente proporcional entre estas variables, de forma que el aumento de la temperatura corporal eleva la percepción subjetiva de esfuerzo. De cara a los resultados de esta investigación, deducimos que la medición de la percepción subjetiva del esfuerzo se presenta como una excelente herramienta para cuantificar y controlar las cargas de una sesión de entrenamiento de fútbol sala.

Palabras clave: Fútbol sala; Temperatura corporal; Percepción subjetiva del esfuerzo; Covid-19; Pandemia.

\section{Introdução}

A pandemia de COVID-19 causada pelo vírus SARS-CoV-2 desencadeou uma emergência global de saúde pública, com rápida evolução e consequências trágicas, com forte impacto nas dimensões social e econômica (Guan et al., 2020; Wu \& Mcgoogan, 2019). O combate a essa doença está em curso, cujas características epidemiológicas, clínicas e prognósticas ainda estão sendo amplamente investigadas por pesquisadores em diferentes partes do mundo (Richardson et al., 2020). Os principais sintomas da doença são febre alta, insuficiência renal e tosse seca. A partir desses sintomas, em nível mundial, iniciou-se a aplicação de protocolos e de medidas sanitárias para se detectar a presença do vírus SARS-CoV-2. Nessa direção, testes salivares passaram a ser uma das formas de se detectar a presença do vírus (Sabino-Silva et al., 2020). Na cidade de Taiwan, foram adotados protocolos de aferição de temperatura corporal, com a finalidade de rastrear pacientes potencialmente portadores da doença COVID-19, caso ultrapassado o limite de $37,5^{\circ} \mathrm{C}$ (Wu \& Chang, 2020). A temperatura corporal elevada se destaca como um importante marcador que sugere a presença da COVID-19, 88\% dos casos diagnosticados com essa doença apresentavam febre (Who 2020a; 2020b; 2020c).

O avanço da doença em escala global exigiu implementação de políticas públicas quanto às medidas sanitárias e isolamento social. Essas medidas geraram restrições à população, obrigando uma série de adaptações no cotidiano das pessoas, 
independentemente de segmentos sociais. Essa demanda não foi diferente para os praticantes de modalidades esportivas, fossem eles amadores ou profissionais, resultando na exigência da aferição de temperatura corporal antes das sessões de treino, bem como do uso de máscara durante essas atividades, com consequentes impactos, tanto na rotina, quanto no desempenho dos atletas. A gravidade da pandemia obrigou ao isolamento social, o que acarretou uma mudança na rotina de treinamento do atleta de futsal, gerando um impacto nas variáveis do desempenho esportivo, específicas no ambiente da prática das modalidades esportivas, entre as quais o futsal (Schmidt \& Wrisberg, 2010). No sentido de minimizar o efeito do isolamento no componente físico do atleta, os jogadores passaram a seguir planos de treinos domiciliares sob a orientação dos treinadores. Embora esses treinamentos permitissem manter um nível de treino adequado em volume e em intensidade, revelavam-se aquém na capacidade de assemelhar as necessidades físicas, técnicas e táticas dos praticantes de modalidades esportivas quando na disputa de um jogo dessa modalidade (Moura et al., 2020).

No futsal o desempenho esportivo é dependente da capacidade de percepção dos praticantes (Travassos, 2021). Os atletas necessitam perceber os sinais relevantes do ambiente, para tomarem uma decisão e agirem face às possibilidades de ação que emergem da interação do sistema indivíduo-ambiente (Gibson, 2014). Portanto, no futsal, o comportamento (ação) dos jogadores é modulado pela interação da tríade indivíduo-tarefa-ambiente (Newell, 1986; Nitsch, 2009). Pelo fato de ser uma modalidade esportiva dinâmica, que exige constantes mudanças de deslocamento e de ações motoras realizadas em intensidades variadas (Soares \& Tourinho, 2006), o futsal gera déficit respiratório e necessidade elevada de trocas gasosas. Em função desse esporte coletivo ser uma atividade acíclica e de caráter intermitente, requer dos salonistas elevados níveis de força, mudanças rápidas de direção e capacidade de realizar deslocamentos em alta intensidade (sprints), repetidos durante as diversas ações do jogo. Como regra, apresenta a possibilidade de realizar um número ilimitado de substituições. Essa possibilidade de substituir infinitamente os praticantes de futsal diminui as oscilações na intensidade durante a partida (Barbero et al., 2008; Gorostiaga et al., 2009).

O jogo de futsal desgasta fisicamente os atletas, desencadeando uma série de mecanismos homeostáticos. O papel mais importante para a autorregulação é a sudorese que, por meio da evaporação do suor pela pele, proporciona a dissipação do calor. Sabe-se que a produção de sudorese é algo variável e individual. Sua intensidade despendida é diretamente associada à temperatura ambiental, bem como a idade, o sexo, performance e estado de hidratação do atleta antes e durante a sessão de treinamento (Carvalho et al., 2011). Esses fatores geram uma oscilação da temperatura corporal e um aumento na percepção subjetiva de esforço (PSE) desenvolvida por Borg (2000).

A escala de PSE é uma forma de avaliação não invasiva, que permite monitorar a intensidade e a percepção de esforço físico (Kaerncher et al., 2018). A aplicação da escala apresenta dois importantes benefícios: a) baixo custo para implementação; b) possibilidade de efetuar relação entre PSE e variáveis fisiológicas, tais como, intensidade do exercício, frequência cardíaca (FC) e consumo de oxigênio (VO2). Com a utilização dessa ferramenta, a identificação mais singular do estado momentâneo, mesmo que de forma subjetiva do atleta, pode auxiliar diretamente que ele atinja um estado de fadiga generalizado (Martín-GuillaumeS et al., 2018).

O processo sistematizado de uma modalidade esportiva tem como objetivo proporcionar mudanças funcionais, morfológicas e metabólicas que impactarão de forma positiva no desempenho dos esportistas (Nakamura et al., 2010). No treinamento desportivo é imperativo conhecer e utilizar ferramentas que auxiliem na prescrição e no controle das cargas individualizadas de treinamento. Esse controle (diagnóstico) possibilita promover alterações no planejamento (prescrição), em função do impacto que as cargas prescritas (carga externa) são atribuídas a cada atleta (carga interna).

No Brasil, considerando o período pandêmico, autoridades sanitárias brasileiras implementaram medidas protetivas a serem aplicadas de forma prévia às sessões de treinamento de equipes de modalidade esportivas. Para atender à legislação brasileira, os clubes, por meio de seus profissionais, passaram a monitorar diariamente seus atletas mediante a mensuração e o 
registro da temperatura corporal. Até o presente momento não há na literatura estudos que investigaram a relação entre a temperatura corporal e a percepção subjetiva de esforço dos praticantes de futsal. Diante dessa realidade, a presente investigação objetivou avaliar as variações de temperatura e de percepção subjetiva de esforço durante as sessões de treinamento de atletas brasileiros praticantes da modalidade futsal.

\section{Metodologia}

\section{Delineamento}

Trata-se de um estudo longitudinal, quantitativo, descritivo, de análise de registros e de documentos (Thomas et al., 2012), o qual visa verificar a variação da temperatura corporal e da percepção subjetiva de esforço - PSE de atletas de futsal de alto rendimento.

\section{Participantes}

A amostra foi composta por 19 atletas do sexo masculino, com idade média de 27,7 $\pm 6,3$ anos, estatura de 1,7 $\pm 0,1$ $\mathrm{m}$, peso de $74,7 \pm 12,8 \mathrm{~kg}$, percentual de gordura de $14,9 \pm 6,6 \%$ e massa magra de $46,3 \pm 5,6 \%$, que participaram assiduamente das sessões de treinamento físico, técnico e tático, realizadas no Ginásio Municipal Jatir Francisco Foresti, localizado na cidade de Marau/RS. As sessões iniciaram-se às 10h (período matutinos) e às 18h30min (períodos noturnos), em quadras de $36 \mathrm{~m}$ por $18 \mathrm{~m}$. Os sujeitos da pesquisa leram e assinaram o Termo de Consentimento Livre e Esclarecido (TCLE. Após a assinatura do termo os dados de temperatura corporal e a PSE foram coletados e registrados em 15 sessões de treinamento realizadas durante a temporada de 2020 (período marcado pela pandemia). A pesquisa obteve a aprovação do Comitê de Ética em Pesquisa em Seres Humanos, da Fundação Universidade de Passo Fundo (CEP), de acordo com os padrões éticos de normas e diretrizes regulamentadoras da pesquisa envolvendo seres humanos, sob o Parecer $\mathrm{n}^{\circ}$ 4.737.074. Todos os procedimentos para a realização da pesquisa foram respeitados, conforme a Resolução 466/2012 do Conselho Nacional de Saúde.

\section{Procedimento}

Os dados coletados foram registrados diariamente. As mensurações da temperatura corporal e da PSE foram realizadas em diferentes momentos do treinamento: a) chegada ao local do treinamento; b) final do aquecimento; c) final da parte principal do treinamento; d) cinco minutos após a sessão do treinamento (avaliando o processo de desaquecimento). Para a verificação da temperatura, foi utilizado um termômetro sensor infravermelho digital de testa, marca Aiqura, com leitura de temperatura de operação entre $0^{\circ} \mathrm{C}$ a $50^{\circ} \mathrm{C}$, com classificação de segurança do laser tipo classe II, com comprimento de onda vermelho entre $635 \mathrm{~nm}$ a $660 \mathrm{~nm}$ e potência de saída menor que $1 \mathrm{~mW}$. Para a verificação da percepção subjetiva de esforço foi utilizada a escala de Borg (2000).

\section{Análise estatística dos dados}

As análises foram realizadas usando o Statistical Package for the Social Sciences (IBM SPSS, versão 25.0). Foi utilizado o teste $t$ de Student para amostras emparelhadas para a comparação das temperaturas, e o teste t de Student para amostras independentes para comparação da PSE. Para verificar se as distribuições eram normais foi aplicado o teste de Shapiro-Wilk. O teste Shapiro-Wilk indicou diferenças significativas em relação à PSE quando se analisou os três momentos de treino (aquecimento, ao final do treino e retorno para casa) para ambos os turnos ( $\mathrm{p}<0,0001)$. Para verificar se as variâncias eram homogêneas foi aplicado o teste de Levene. Adotou-se um nível de significância de $\mathrm{p} \leq 0,05$ para rejeitar a hipótese nula, para um intervalo de confiança de $95 \%$. 


\section{Resultados}

A Figura 1 demonstra a variação da temperatura corporal aferida nos diferentes momentos do treinamento, discriminando os turnos da manhã e noite.

Figura 1. Médias da variação de temperatura corporal por turno, em ${ }^{\circ} \mathrm{C}$, nos diferentes momentos das sessões de treino.

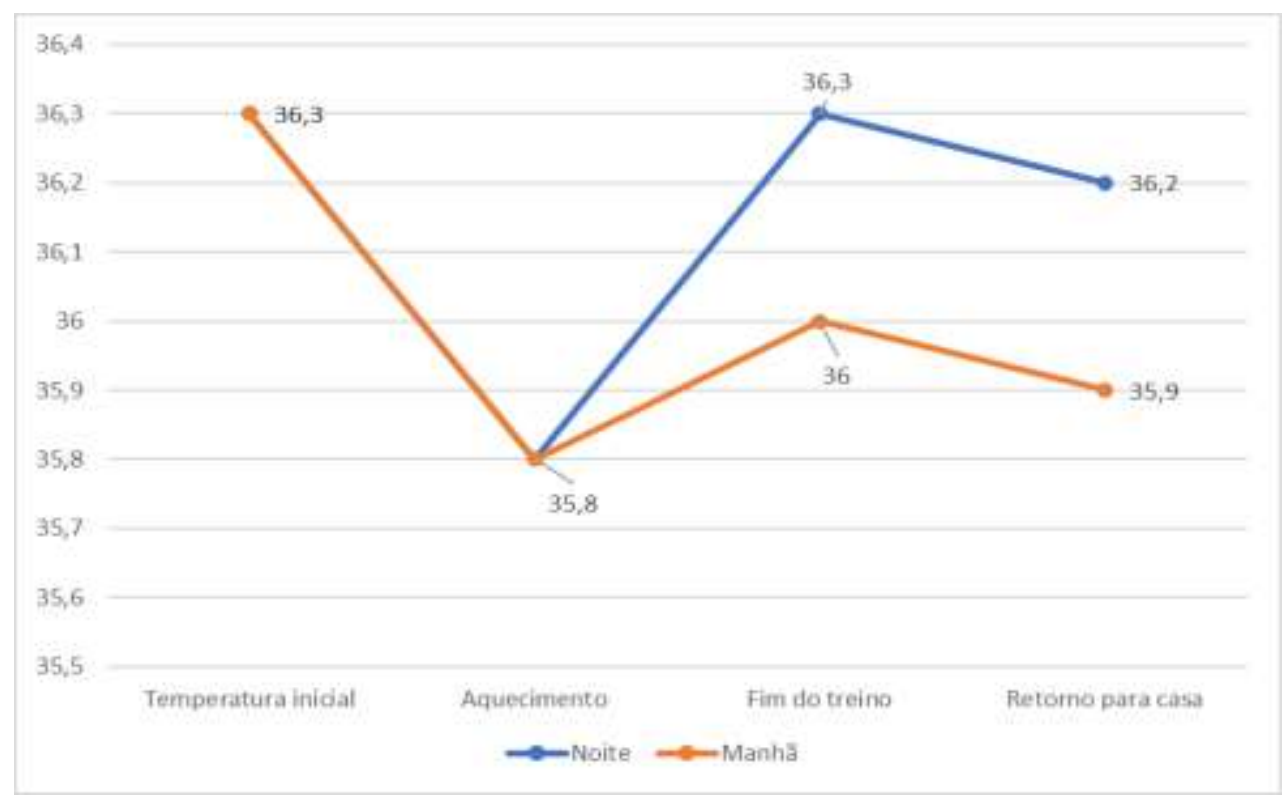

Fonte: Autores.

Ocorreu uma variação entre $35,8^{\circ} \mathrm{C}$ e $36,3^{\circ} \mathrm{C}$. A média da temperatura ambiente nas sessões de treino foi de $22,6 \pm$ $1,8^{\circ} \mathrm{C}$. A temperatura de chegada dos atletas na sessão de treinamento no turno da manhã foi, em média, de $36,3 \pm 0,5^{\circ} \mathrm{C}$, passando para $35,8 \pm 0,5^{\circ} \mathrm{C}$ no final do aquecimento, sendo registrado $36 \pm 0,7^{\circ} \mathrm{C}$ no final do treinamento e $36 \pm 0,7^{\circ} \mathrm{C}$ na saída de retorno para casa. Já no turno da noite a temperatura de chegada dos atletas na sessão de treinamento no turno da manhã foi, em média, de $36,3 \pm 0,5^{\circ} \mathrm{C}$, passando para $35,8 \pm 0,6^{\circ} \mathrm{C}$ no final do aquecimento, sendo registrado $36,2 \pm 0,6^{\circ} \mathrm{C}$ no final do treinamento e $36,2 \pm 0,7^{\circ} \mathrm{C}$ na saída de retorno para casa. A temperatura no final do treino da noite é mais elevada do que na sessão de treino realizada pelo turno da manhã. Este fator pode estar relacionado diretamente à intensidade proposta nas sessões de treino, onde um repertório de ações mais intensas pode elevar a temperatura, mais do que atividades de menor intensidade. A Figura 2 apresenta as médias de temperaturas por turno, nos diferentes momentos de coletas. 
Figura 2. Demonstrativo das médias de temperaturas por turno, nos diferentes momentos de coletas.

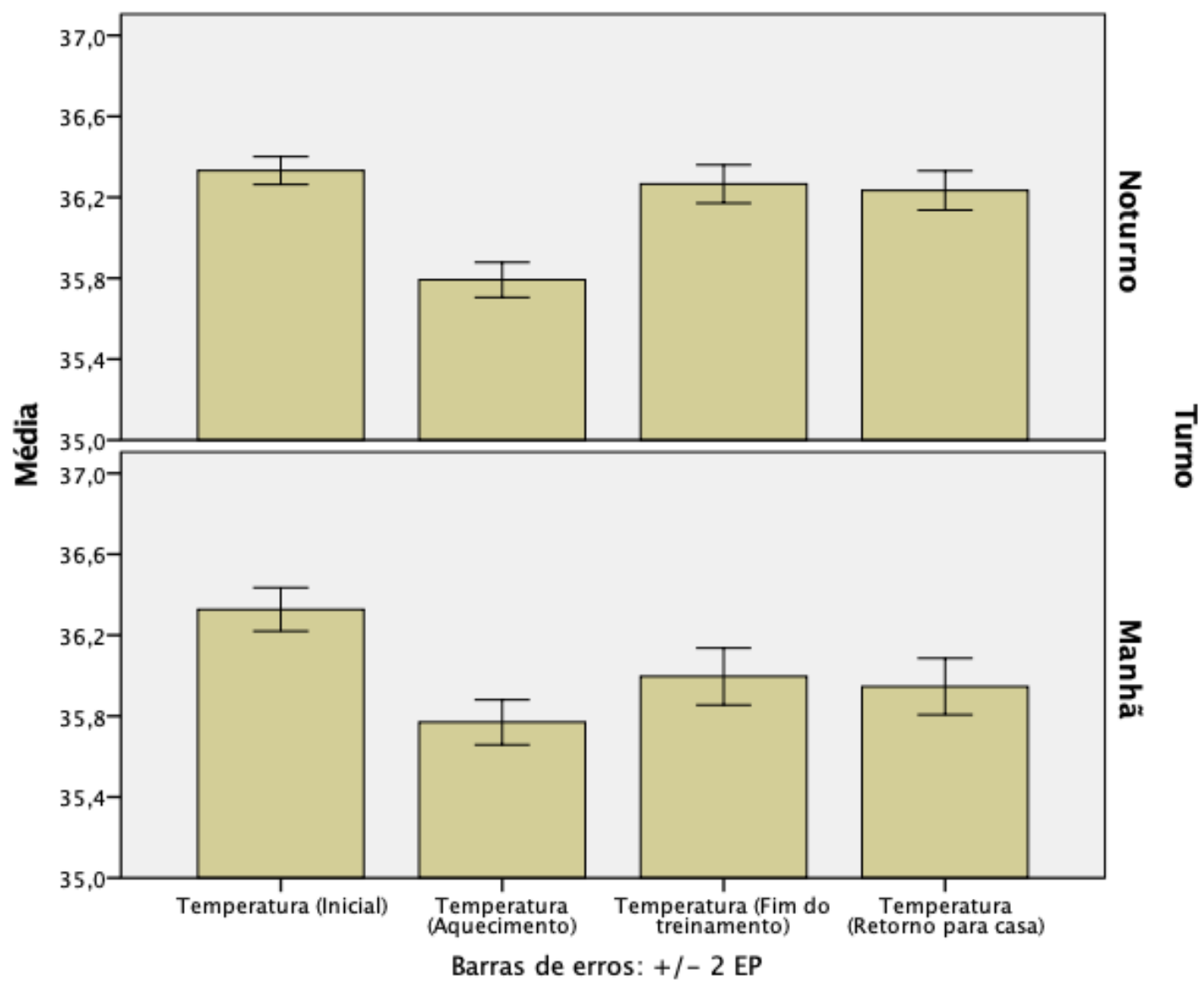

Fonte: Autores.

Observa-se uma diferença significativa entre a temperatura inicial e a temperatura pós-aquecimento, no turno da manhã ( $\mathrm{p}<0,001)$, temperatura do fim do treino e retorno para casa, no turno da noite a diferença ocorreu somente entre a temperatura inicial e o pós aquecimento $(\mathrm{p}<0,001)$. A Figura 3 demonstra as médias das temperaturas por tuno, no final do treinamento. 
Research, Society and Development, v. 11, n. 2, e38611225957, 2022

(CC BY 4.0) | ISSN 2525-3409 | DOI: http://dx.doi.org/10.33448/rsd-v11i2.25957

Figura 3. Médias das temperaturas por turno, em ${ }^{\circ} \mathrm{C}$, no final do treinamento.

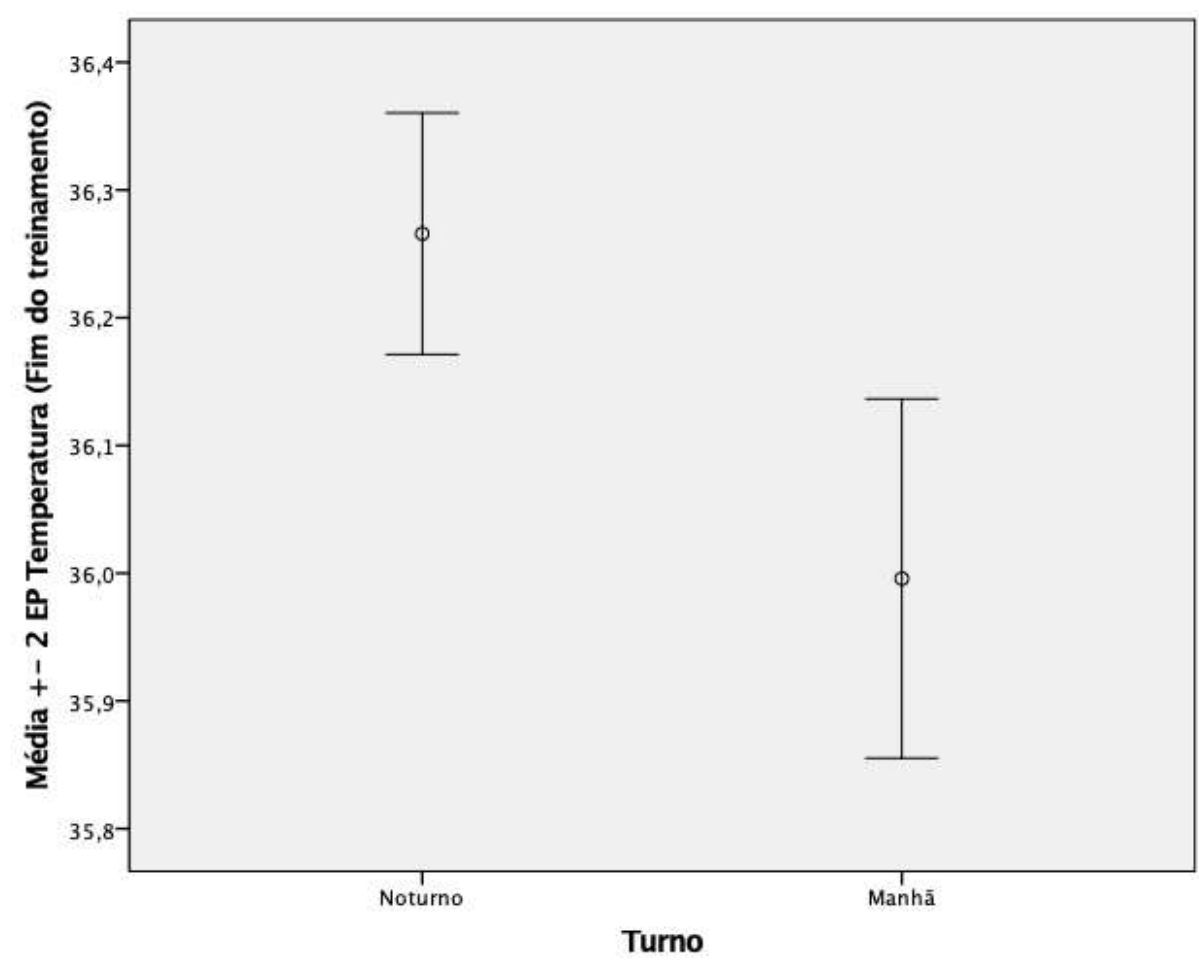

Fonte: Autores.

Ocorreu uma maior temperatura no turno da noite. Esse indicativo pode estar relacionado diretamente a um possível acúmulo de desgaste físico gerado pela sessão anterior (turno da manhã) e que o tempo proposto de intervalo ente as sessões (6h30min) não seja o tempo suficiente para a plena recomposição do indivíduo. O tempo de intervalo da sessão noturna para a sessão da manhã é composta pelo descanso, repouso e sono do atleta com um intervalo maior do que o tempo entre as sessões no mesmo dia, o que pode auxiliar diretamente na recuperação dele. A Figura 4 apresenta as médias de temperatura por turno, em ${ }^{\circ} \mathrm{C}$, no retorno para casa. 
Research, Society and Development, v. 11, n. 2, e38611225957, 2022

(CC BY 4.0) | ISSN 2525-3409 | DOI: http://dx.doi.org/10.33448/rsd-v11i2.25957

Figura 4. Médias das temperaturas por turno, em ${ }^{\circ} \mathrm{C}$, no retorno para casa.

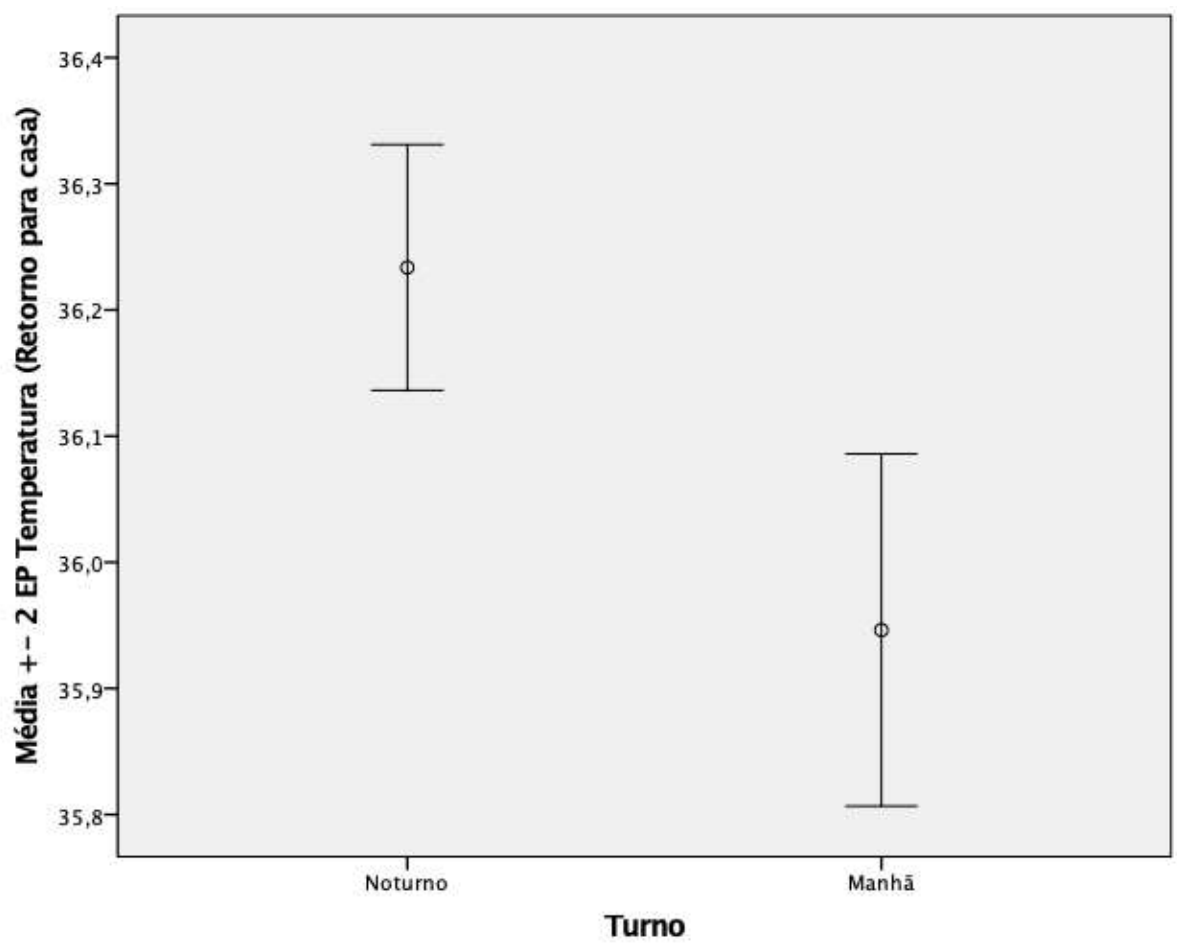

Fonte: Autores.

Ocorreu uma maior elevação da temperatura corporal dos atletas no momento do retorno para casa no turno da noite quando se compara com a temperatura média aferida no turno da manhã. A Figura 5 apresenta as médias da PSE por turno, aferidas nos diferentes momentos das sessões de treinamento. 
Figura 5. Médias da percepção subjetiva de esforço por turno durante o aquecimento, ao final do treino e no momento de retorno para casa.

\section{Turno}

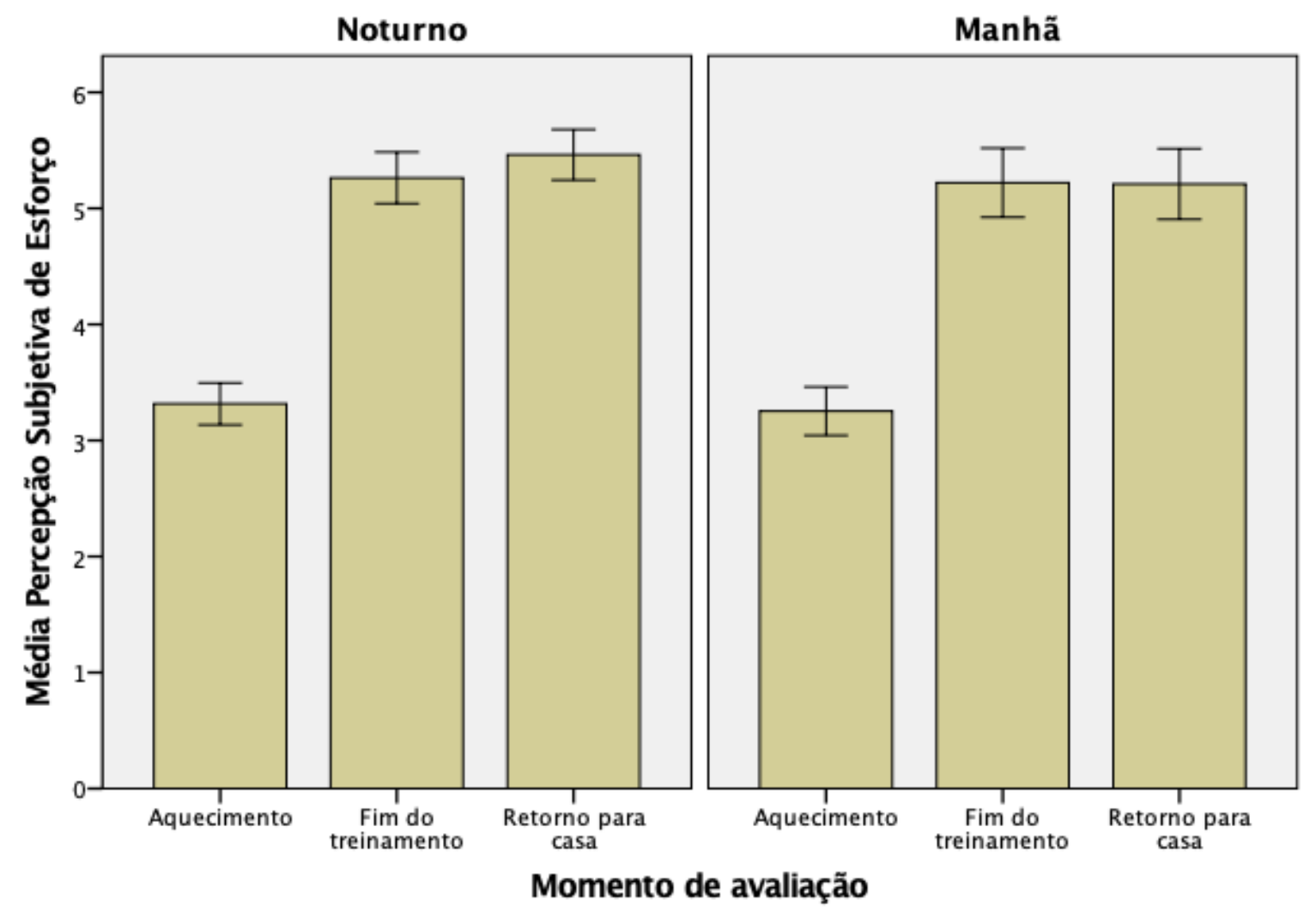

Barras de erros: $+/-2$ EP

Fonte: Autores.

No início do treinamento da manhã, após o aquecimento, manteve-se em uma média de 3,25 $\pm 1,02$, passando para $5,22 \pm 1,45$ no final do treino, e 5,21 $\pm 1,48$ no retorno para casa, enquanto no turno da noite as médias foram de 3,32 $\pm 1,24$, passando para 5,26 $\pm 1,52$ e 5,46 $\pm 1,50$ respectivamente, conforme o treino foi acontecendo. As diferenças ocorreram tanto no turno da manhã, como no da noite, comparando o fim do treinamento e o retorno para casa, com a medida de pós aquecimento apresentando diferença significativa $(\mathrm{p}<0,001)$. Os achados demonstram que a média da PSE no final do treino da noite $(5,26$ $\pm 1,52)$ e no retorno para casa $(5,46 \pm 1,50)$ foram superiores às detectadas no turno da manhã. A intensidade empregada, o intervalo entre as sessões (manhã/noite) ou o acúmulo de ações realizadas individualmente por cada atleta durante o dia podem gerar fatores que interferem diretamente nesta elevação. A Figura 6 apresenta as médias da PSE nos diferentes momentos do treino por turno. 
Figura 6. Médias da PSE nos diferentes momentos do treino por turno.

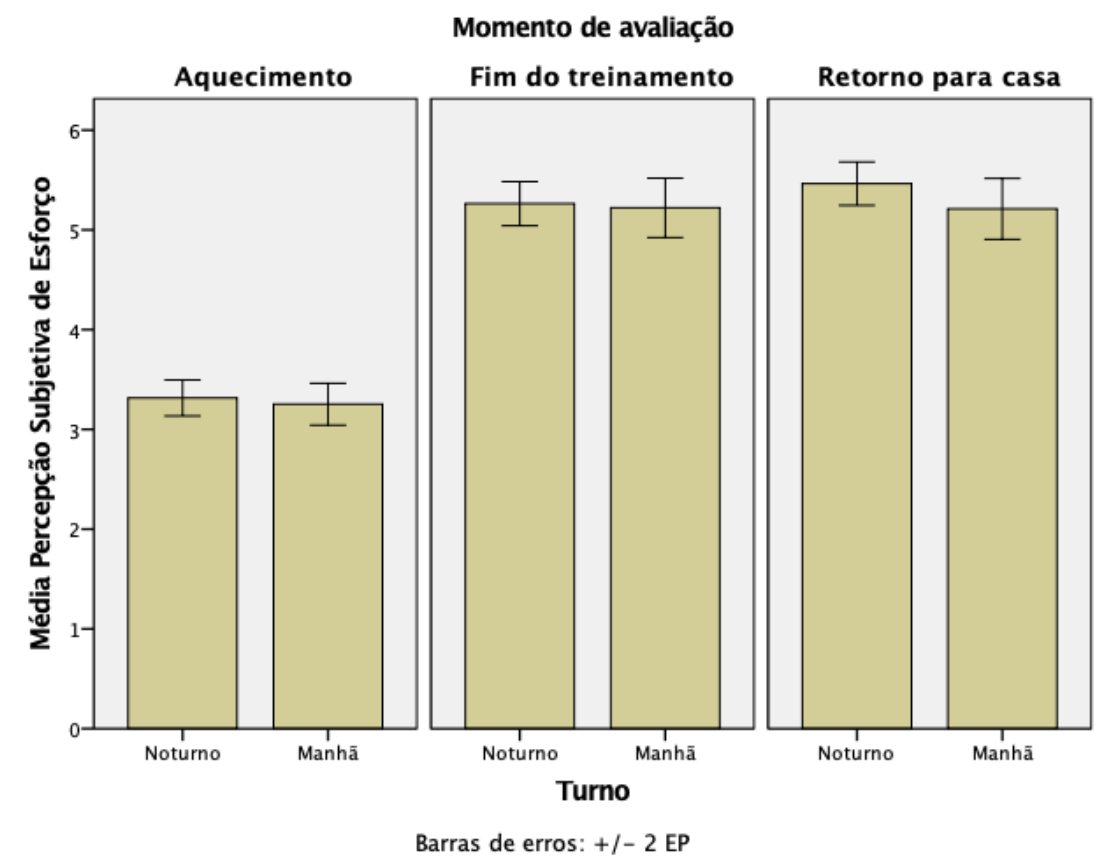

Fonte: Autores.

Percebe-se uma relação entre a elevação da temperatura corporal e a elevação da PSE, demonstrando que, quanto maior a elevação de temperatura corporal como uma resposta fisiológica do atleta, a sua PSE se eleva naturalmente, podendo assim ser usada como um parâmetro sólido de indicação para tais alterações. A PSE de retorno para casa, se manteve alta, em especial a do turno da noite, comparada com o final da sessão de treinamento, deixando claro uma necessidade maior de intervalo entre esses dois momentos, bem como a necessidade de um processo de desaceleração ou desaquecimento nos póstreinos, reduzindo essa percepção do atleta e auxiliando no seu pronto restabelecimento, seja entre turno manhã noite, seja noite/manhã.

\section{Discussão}

A presente pesquisa objetivou analisar as variações de temperatura corporal e de Percepção Subjetiva de Esforço durante as sessões de treinamento no futsal, bem como descrever a relação entre as duas variáveis. Em jogos oficiais de futsal, os atletas atingem entre $86-90 \%$ da frequência cardíaca máxima (FCmax) e 76 e $79 \%$ do consumo máximo de oxigênio (VO2max) (Barbero et al., 2008; Castagna et al., 2009; Rodrigues et al., 2011).

Assim, o futsal é caracterizado como um esporte intermitente de alta intensidade e com elevada solicitação do metabolismo aeróbio. No entanto, a modalidade também exige significativa participação do metabolismo anaeróbio, em função do elevado número de ações intermitentes de alta intensidade ou de sprints múltiplos, tais como acelerações, desacelerações e sprints realizadas durante os jogos (Barbero \& Andrín, 2005; Barbero et al., 2008; Castagna et al., 2009). Esses fatores aceleram o metabolismo do atleta por gerar uma elevação na frequência cardíaca e, consequentemente, um aumento na temperatura corporal. A transpiração tem a função da termorregulação e é responsável pelo ajustamento da temperatura corporal, tendo como função o equilíbrio entre a produção (termogênese) e a dissipação (termo dispersão) do calor central para manter a temperatura corporal interna em aproximadamente $36,5^{\circ} \mathrm{C}$ (Prado et al., 2010).

No presente estudo, observou-se uma variação na temperatura corporal do momento da chegada na sessão de treinamento $\left(\right.$ manhã $=36,3 \pm 0,52^{\circ} \mathrm{C}$, e noite $=36,3 \pm 0,47^{\circ} \mathrm{C}$ ), até o final da sessão de treino, no entanto essa elevação não foi 
significativa. Por outro lado, a média da temperatura verificada no turno da noite foi significativamente maior do que a média do turno da manhã. Assim, quando o corpo humano é exposto à quebra de seu estado de repouso, ocorre como resultado uma resposta aguda (aumento da produção de calor) e, consequentemente, ativação dos mecanismos fisiológicos de controle da temperatura e de hidratação. Quando essa exposição ganha intensidade, esse parâmetro se torna extremamente relevante, assim limitando a continuidade desta atividade (Cardoso \& Souza 2010).

Durante essa exposição, o calor é um subproduto gerado pelo próprio metabolismo, elevando a temperatura corporal, porém, a elevação deverá ser mantida de maneira estável, próximo aos $37^{\circ} \mathrm{C}$, utilizando-se de mecanismos neurais e cardiovasculares (Guyton \& Hall, 2015). Durante a prática de exercícios físicos, a oscilação da temperatura muscular pode aumentar até $2^{\circ} \mathrm{C}$, em comparação com a temperatura de repouso, sendo que a temperatura central do corpo pode atingir até $39^{\circ} \mathrm{C}$. No entanto, algumas variáveis, como tipo de esporte, características de regras e regulamentos, fatores exógenos, têm um impacto significativo sobre as possibilidades de hidratação do praticante, podendo dificultar o resfriamento corporal (Godois et al., 2014).

Na modalidade em questão, e para a amostra analisada, a elevação da temperatura não chegou a tal índice, o que pode estar associado às frequentes paradas para recuperação e hidratação do atleta, práticas comuns nas sessões de treinamento do futsal, evitando a fadiga precoce e a queda de rendimento do atleta na sessão de treino e consequentemente a baixa elevação da temperatura. Essas paradas frequentes são baseadas na fisiologia corporal, buscando controlar a intensidade do exercício ou, mais especificamente, o estresse que ocorre sobre os sistemas fisiológicos periféricos, tais como o sistema cardiopulmonar e o sistema muscular (Pinheiro et al., 2014). Os preparadores físicos e fisiologistas utilizam alguns métodos para o controle das intensidades, risco de fadiga e lesão. Segundo Esposito et al. (2004) a PSE pode ser um indicador válido da intensidade de exercício. Para Noakes (2004) a PSE é uma importante ferramenta para garantir que o exercício seja executado dentro de limites seguros, sem a ocorrência de danos ao atleta. Em pesquisa realizada por Alexiou e Coutts (2008), os autores observaram fortes correlações entre o método da PSE da sessão e os impulsos do treino (TRIMP) pelos métodos de frequência cardíaca $(r=0,83$ e 0,85), comparáveis às observadas em modalidades cíclicas e aeróbias.

Ao avaliar atletas da categoria sub-20 em um jogo simulado com duração total de 80 minutos, os resultados de uma pesquisa de Arruda e et al. (2013) mostraram uma variação nos scores de 5 a 6, classificando o experimento como difícil a muito difícil. Na pesquisa de Silva e et al. (2015), os scores encontrados em jogos de futsal foram valores medianos de PSE = 7, sendo classificado como vigoroso. Analisando o intervalo interquartil (4,0 - 7,5), nota-se que os valores não ultrapassam o limite de intensidade leve, apenas o da intensidade moderada. No estudo de Santa-Cruz et al. (2016), foram encontrados valores médios de 7,5 e 7,4 para as partidas da $1^{a}$ fase e 8,2, 8,7 e 8,9 para os jogos de futsal da fase final, indicando cargas crescentes ao longo da competição.

\section{Considerações Finais}

No presente estudo a PSE demonstrou diferença significativa no desenrolar do treino, com uma elevação da percepção do atleta, conforme o treino avançava tanto no turno da manhã, quanto no turno da noite. Outro fator relevante, foi que a média da PSE encontrada no turno da noite foi significativamente maior do que a média encontrada no turno da manhã. Esses achados estão abaixo dos encontrados nos estudos citados, que se referem diretamente a PSE coletada no transcorrer dos jogos, e não dos treinamentos. Assim como a temperatura corporal, que não apresentou uma elevação, a PSE de treinamento pode também ter apresentado escores mais baixos, devido as pausas repentinas realizadas para evitar a extenuação dos atletas, apresentando uma paridade entre si. Considerando esses aspectos, o controle da dinâmica de carga/recuperação é um fator necessário para o desempenho físico no esporte, sendo imprescindível dar igual importância tanto para o treinamento, quanto para a recuperação (Laurent et al, 2011). Ressalta-se que o método da PSE coletado durante uma sessão de treinamento 
apresenta alta fidedignidade para quantificar cargas de treinamento, apoiando-se em outros estudos e com os achados da presente pesquisa, que retratou uma relação de alta correlação da elevação da PSE, conforme elevava a temperatura corporal e a intensidade ofertada no treino.

O método PSE pode ser usado como boa estratégia para o controle de cargas de treinamento de atletas de futsal de alto rendimento masculino. O presente estudo buscou a relação da temperatura corporal e da PSE durante a sessão de treino, como uma iniciativa inovadora, que permitiu relacionar as duas variáveis, com alto teor de equivalência em um período de 15 sessões de treinamento. Esse fato contribui com a variável temperatura corporal como um indicador válido, fidedigno e objetivo que pode servir como mais um aliado importante no momento de avaliação, prescrição e controle das sessões de treino. Isso permite, aos membros da comissão técnica de uma equipe de futsal, monitorar adaptações agudas ocorridas durante o treinamento em atletas de futsal.

O futsal é uma modalidade que vem crescendo constantemente e exige dos profissionais envolvidos nas comissões técnicas um aprendizado constante, buscando sempre qualificar suas intervenções por meio de sessões representativas de treino e, ao mesmo tempo, prescrever cargas externas, respeitando as restrições estruturais e funcionas de cada atleta. A atividade de repouso ativo como trote contendo mudanças de direção em velocidades abaixo de $5 \mathrm{~km} / \mathrm{h}$, alongamentos ativos e passivos, liberação mio facial e massagem relaxante, podem ser alternativas que auxiliem na recuperação do atleta pós treino, minimizando os efeitos inflamatórios gerados pela sessão de treino, diminuindo assim os riscos de lesão e a fadiga precoce do atleta.

Os autores sugerem novos estudos voltados ao uso dessas ferramentas durante as sessões de treinamento, bem como o cruzamento com outras, que possam contribuir na avaliação, na prescrição e no controle das cargas de treino, com o objetivo de melhorar o desempenho esportivo e a vida útil do atleta.

\section{Referências}

Alexiou, H., \& Coutts, A. J. (2008). A comparison of methods used for quantifying internal training load in women soccer players. International Journal of Sports Physiology and Performance, 3 (3), 320-330. https://doi.org/10.1123/ijspp.3.3.320

Arruda, A. F. S., Aoki, M. S., Freitas, C. G., Coutts, C., \& Moreira, A. (2013). Planejamento e monitoramento da carga de treinamento durante o período competitivo no basquetebol. Revista Andaluza de Medicina del Deporte, 6 (2), 85-89. https://doi.org/10.1016/S1888-7546(13)70040-6

Barbero, J. C. B. A., \& Andrín, G. (2005). Desarrollo y aplicación de um nuevo test de campo para resistência específica em jugadores de fútbol sala: TREIF (teste de resistência específica intermitente para futsal). Efdeportes.com Revista Digital, 89, 1-6. https://dialnet.unirioja.es/servlet/articulo?codigo=1285154

Barbero, J. C. A., Soto, V. M., Barbero, V. A., \& Granda- Vera, J. (2008) Match analysis and heart rate of futsal players during competition. Journal of Sports Sciences, 26 (1), 63-73. https://doi.org/10.1080/02640410701287289

Borg, G. (2000). Escalas de Borg para a Dor e Esforço Percebido. Editora Manole.

Cardoso, S., \& Souza, C. (2010). Aspectos fisiológicos da ingestão de água no organismo humano e sua influência no rendimento atlético. Revista de trabalhos acadêmicos, 1, 1-9. https://pt.slideshare.net/Dayrinha/aspectos-fisiolgicos-da-ingesto-de-gua-no-organismo-humano

Carvalho, H. A., Silva, R. P., Belfort, F. G., \& Moreira. O. C. (2011). Avaliação do equilíbrio hídrico em estudantes do ensino médio submetidos a jogos de futsal durante a aula de educação física. Revista Brasileira de Futebol 4 (2), 41-48. https://rbf.ufv.br/index.php/RBFutebol/article/view/89

Castagna, C., D'Ottavio, S., Granda Vera, J., \& Barbero Alvarez, J. C. (2009). Match demands of professional Futsal: a case study. Journal of science and medicine in sport, 12 (4), 490-494. https://doi.org/10.1016/j.jsams.2008.02.001

Esposito, F., Impellizzeri, F. M., Margonato, V., Vanni, R., Pizzini, G., \& Veicsteinas, A. (2004). Validity of heart rate as an indicator of aerobic demand during soccer activities in amateur soccer players. European journal of applied physiology, 93(1-2), 167-172. https://doi.org/10.1007/s00421-004-1192-4

Gibson, J. J. (2014). The ecological approach to visual perception: classic edition. Psychology Press

Godois, A. M., Raizel, R., Behrends, V., Fabrício, R., Ravagnani, C. P., Fett, C. A., Voltarelli, F. A., \& Ravagnani, C. F. C. (2014). Perda hídrica e pratica de hidratação em atletas de futebol. Revista Brasileira de Medicina do Esporte, 20 (1), 47-50. https://doi.org/10.1590/S1517-86922014000100009

Gorostiaga, E. M., Llodio, I., Ibáñez, J., Granados, C., Navarro, I., Ruesta, M., Bonnabau, H., \& Izquierdo, M. (2009). Diferences in physical Wtness among indoor and outdoor elite male soccer players. European Journal of Applied Physiology, 106 (4), 483-491. https://doi.org/10.1007/s00421-009-1040-7 
Guan, W., Ni, Z., Hu, Y., Liang, W., Ou, C., He, J. et al. (2020). Clinical Characteristics of Coronavirus Disease 2019 in China. The new england journal of medicine, $382(8), 1708-1720$.

Guyton, A. C., \& Hall, E. J. (2015). Guyton and hall textbook of medical physiology. Philadelphia: Elsevier Saunders.

Kaerncher, P. L. K., Glänzel, M. H., Rocha, G. G. d., Schmidt, L. M., Nepomuceno, P., Stroschöen, L., \& Reckziegel, M. B. (2018). Escala de percepção subjetiva de esforço de Borg como ferramenta de monitorização da intensidade de esforço físico. RBPFEX. Revista Brasileira de Prescrição e Fisiologia do Exercício, 12 (80) (Sup. 3). https://www.rbpfex.com.br/index.php/rbpfex/article/view/1603

Laurent, C. M., Green, J. M., Bishop, P. A., Sjökvist, J., Schumacker, R. E., Richardson, M. T., \& Curtner-Smith, M. (2011). A practical approach to monitoring recovery: development of a perceived recovery status scale. Journal of strength and conditioning research,25 (3), 620-628. https://doi.org/10.1519/JSC.0b013e3181c69ec6

Martín-Guillaumes, J., Caparrós, T., Cruz-Puntí, D., Montull, L., Orriols, G., \& Capdevila, L. (2018). Monitorización psicofisiológica del proceso de recuperación en deportistas de élite de la selección española de esquí de montaña a través de la rmssd y la percepción subjetiva de la recuperación. Revista Iberoamericana de Psicología del Ejercicio y El Deporte. 13 (2), 219-223. https://dialnet.unirioja.es/servlet/articulo?codigo=6543404

Moura, L. D., Dias, A., Torre, J. P., Farinha, P., Ribeiro, B., \& Cordeiro, C. R. (2020). Pandemia covid-19 e Impacto no Desporto. Revista Medicina Desportiva Informa, 11 (3), 26-33.

Nakamura, F. Y., Moreira, A., \& Aoki, M. S. (2010). Monitoramento da carga de treinamento: a percepção subjetiva do esforço de uma sessão é um método confiável? Jornal de Educação Física, 21 (1), 1-11. https://doi.org/10.4025/reveducfis.v21i1.6713

Newell, K. M. (1986). Constraints on the development of coordination. In M. G. Wade \& H. T. A. Whiting (Eds.), Motor skill acquisition in children: Aspects of coordination and control (pp. 341-360). Nato Cientific.

Noakes, T. D. (2004). Linear relationship between the perception of effort and the duration of constant load exercise that remains. Journal of Applied Physiology, 96 (4), 1571-1573. https://doi.org/10.1152/japplphysiol.01124.2003

Nitsch, J. R. (2009). Ecological approaches to Sport Activity: A commentary from an actiontheoretical point of view. Int. J. Sport Psychol., 40 (1),152-176.

Pinheiro, F. A., Viana, B., \& Pires, F. O. (2014). Percepção subjetiva de esforço como marcadora da duração tolerável de exercício. Motricidade. 10 (2), 100106. https://doi.org/10.6063/motricidade.10(2).2267

Prado, E. S., Gonzaga, W. S., \& Dantas, E. H. M. (2010). Conhecimento das práticas de hidratação dos atletas de vôlei de praia do estado de Sergipe. Revista Brasileira de Ciência e Movimento, 18 (3), 29-34.

Richardson, S., Hirsch, J. S., Narasimhan, M., Crawford, J. M., McGinn, T., Davidson, K. W. et al. (2020). Presenting Characteristics, Comorbidities, and Outcomes Among 5700 Patients Hospitalized With COVID-19 in the New York City Area. JAMA, 323 (20), 2052-2059. https://doi.org/10.1001/jama.2020.6775

Rodrigues, V. M., Ramos, G. P., Mendes, T. T., Cabido, C. E. T., Melo, E. S., Condessa, L.A., Coelho, D. B., Garcia, E. S. (2011). Intensity of official futsal matches. Journal of Strength and Conditioning Research, 25 (9), 2482-7. https://doi.org/10.1519/JSC.0b013e3181fb4574

Sabino-Silva, R, Jardim, A. C. G., \& Siqueira, W. L. (2020). Coronavirus covid-19 impacts to dentistry and potential salivary diagnosis. Clinical oral investigations, 24 (4), 1619-1621. https://doi.org/10.1007/s00784-020-03248-x

Santa-Cruz, R. A. R., Campos, F. A. D., Gomes, I. C. B., \& Pellegrinotti, I. L. (2016). Percepção subjetiva do esforço em jogos oficiais de Futsal. Revista Brasileira de Ciência e Movimento, 24 (1), 80-85. http://dx.doi.org/10.18511/rbcm.v24i1.5813

Schmidt, R. A., \& Wrisberg, C. A. (2010). Aprendizagem e performance motora: iniciando. Artmed.

Silva, D. H. F., Oliveira, D. M., Oliveira, L., Alves, N. S., \& Coledam, D. H. C. (2015). Comparação entre a intensidade do esforço do jogo de futsal com o exergame em adolescentes. Anais do I Simpósio Interdisciplinar de Tecnologias na Educação [SInTE] - IFSP, Câmpus Boituva 24 a 26 de junho de 2015 Boituva SP.

Soares, B., \& Tourinho, F. H. (2006). Análise da distância e intensidade dos deslocamentos, numa partida de futsal, nas diferentes posições de jogo. Revista Brasileira de Educação Física Esportiva, 20 (2), 93-101. https://doi.org/10.1590/S1807-55092006000200002

Thomas, J. R., Nelson, J. K., \& Silverman, S. J. (2012)Métodos de pesquisa em atividade física. (6a ed.), Artmed.

Travassos, B. (2021). Desenho e manipulação de exercícios de treino no futsal: da conceitualização à prática. Secco Editora.

WHO - World Health Organization. Report of the WHO-China Joint Mission on Coronavirus Disease 2019 (Covid-19). https://www.who.int/docs/defaultsource/coronaviruse/who-china-joint-mission-on-covid-19-final-report.pdf.

WHO - World Health Organization. Coronavirus disease 2019 (covid-19). https//www.who.int/docs/default-source/coronaviruse/situation-reports/20200226sitrep-37-covid-19.pdf

WHO - World Health Organization. Novel Coronavirus (2019-nCoV). https://www.who.int/emergencies/diseases/novel-coronavirus-2019/situation-reports. 2020c.

Wu, M., Chang, Y. C. (2020). Covid-19 and its implications in the management of resource infrastructure. Journal of Dental Sciences, 15 (2), $225-226$. https://doi.org/10.1016/j.jds.2020.04.008

Wu, Z., \& McGoogan, J. M. (2020). Characteristics of and Important Lessons From the Coronavirus Disease 2019 (COVID-19) Outbreak in China: Summary of a Report of 72314 Cases From the Chinese Center for Disease Control and Prevention. JAMA, 323 (13), 1239-1242. https://doi.org/10.1001/jama.2020.2648 